\title{
DSM-5: what is new and what is next?
}

\author{
DSM-5: o que é novo e o que vem por aí?
}

Luis A. Rohde, ${ }^{1}$ Marcia Kauer-Sant'Anna ${ }^{2}$

The DSM-5 came out after all. It came with important changes, but with less innovation than some would expect. In the beginning of the review process, the DSM5 was announced as a paradigm change in psychiatry, by linking diagnosis to pathophysiology. ${ }^{1}$ However, the neurobiological findings available were not robust enough to allow inclusion in the classification system. Also, any proposed modification was carefully examined to protect psychiatric diagnosis, keeping a balance between sensitivity and specificity of diagnoses in psychiatry, and also to avoid deleterious consequences of any changes to criterion validity. ${ }^{2}$ However, classifications in psychiatry still lack predictive value and are not consistently associated with a causative explanation of phenomena in comparison with other areas of medicine.

Despite the caveats inherent to any classification system, the importance of the DSM-5 is undeniable, compiling information and facilitating clear communication in clinical and research fields. The major changes in DSM-5 classification were: a) focus on dimensional and developmental perspectives (in all disorders rather than only in a subgroup previously categorized as diagnoses first evidenced in childhood and adolescence); b) reordering of disorders and new grouping based on shared etiological factors; c) abandonment of the multi-axial structure; d) changes in existing diagnostic criteria for several disorders (after 5 years of evidence review); e) new diagnoses (i.e., hoarding disorders, disruptive mood dysregulation disorder (DMDD), binge eating disorder, premenstrual dysphoric disorder); and f) a chapter on disorders that need further study, e.g., attenuated psychosis syndrome and non-suicidal self-injury.

Among the new diagnoses, DMDD is perhaps the most remarkable and controversial entity. Noteworthy, it is included in the depressive disorders chapter, calling attention to the fact that most children with DMDD develop major depressive disorder (not bipolar disorder) or generalized anxiety disorder when adults. ${ }^{3}$ The rationale behind this new diagnostic category takes into account the debate that rates of bipolar disorder in children and adolescents have increased much faster than in adults. ${ }^{4}$ Some studies have attributed such high rates to changes in diagnostic practices, with children who lack traditional manic symptoms being diagnosed as bipolar on the basis of alternative symptoms - especially irritability. ${ }^{5}$ In this context, the diagnosis of severe mood dysregulation (SMD) emerged a decade ago ${ }^{6}$ as a differential diagnosis for bipolar disorder, given their different courses in adulthood. The DMDD diagnosis includes children and adolescences who present with hyperarousal along with irritability and frequent temper outbursts. $^{7}$

Of all changes made to existing diagnostic criteria, two are of particular impact: the elimination of mixed episodes from the bipolar disorders chapter and the modification of age of onset for attention-deficit/hyperactivity disorder (ADHD). Instead of mixed episodes, now 'with mixed features' is a possible specifier of any mood episode, recognizing the fact that there should always be a predominant polarity, with or without symptoms of the opposite pole (mixed features). This change brings

\footnotetext{
${ }^{1}$ Department of Psychiatry, Universidade Federal do Rio Grande do Sul (UFRGS), Porto Alegre, RS, Brazil. Institute for Developmental Psychiatry for Childhood and Adolescence, Brazil. ${ }^{2}$ Bipolar Disorder Program and Laboratory of Molecular Psychiatry, National Science and Technology Institute for Translational Medicine (INCT-TM), Conselho Nacional de Desenvolvimento Científico e Tecnológico (CNPq), Hospital de Clínicas de Porto Alegre (HCPA), Faculdade de Medicina, Universidade UFRGS, Porto Alegre, RS, Brazil.

Conflicts of interest: Luis A. Rohde was on the speakers' bureau and/or acted as a consultant for Eli-Lilly, Janssen-Cilag, Novartis, and Shire over the last 3 years. He also received travel awards (air tickets and hotel costs) from Novartis and Janssen-Cilag in 2010 for taking part of two child psychiatric meetings. The ADHD and Juvenile Bipolar Disorder Outpatient Programs chaired by Luis A. Rohde received unrestricted educational and research support from the following pharmaceutical companies in the last 3 years: Abbott, Eli-Lilly, Janssen-Cilag, Novartis, and Shire. Marcia Kauer-Sant'Anna is on the speakers'/advisory boards for, or has received research grants from NARSAD, Stanley Medical Research Institute, CNPq-Universal, CNPq/INCT-TM, FIPE-HCPA, and Eli-Lilly. Suggested citation: Rohde LA, Kauer-Sant'Anna M. DSM-5: what is new and what is next? Trends Psychiatry Psychother. 2013;35(3):149-50.
} 
criteria closer to what was observed in clinical practice and improves the diagnosis of mixed features, with great implications for treatment approach. In ADHD, the DSM-5 now requires that symptoms should be present before 12 years of age (not anymore 7 years of age, as in the DSMIV). Several studies found no differences in phenotypic presentation, neuropsychological impairment, course, severity, or treatment response between children with onset of symptoms before or after 7 years of age. ${ }^{8}$ The medical and general community have voiced concerns that such a change may artificially increase the prevalence of ADHD, but evidence from population studies does not support this claim. ${ }^{9}$

A number of other changes were made to the DSM-5, and reviewing them is beyond the scope of this editorial. Complete reviews have been published elsewhere. ${ }^{1}$ In this issue of Trends in Psychiatry and Psychotherapy, an important article from DeSousa et al. provides methodological support to further studies on the new diagnosis proposed in the DSM-5, namely DMDD. ${ }^{10}$ This is highly warranted, and will contribute to the understanding of the clinical value of this new diagnostic category in different cultures.

As per the DSM-5 preface, it was designed first and foremost to be a useful guide to clinical practice. And, as an official nomenclature, it must be applicable in a wide diversity of contexts. ${ }^{11}$ The DSM may also be a tool for collecting and communicating accurate mental health statistics. After all, in our view, it has achieved the goal of producing the best available evidence-based tool for the classification and description of mental disorders. There is always room for enhancement and updating.

Even DSM-5 limitations have contributed to the acknowledgement that, in fact, the field of psychiatry has produced only modest progress over the last years. Clinical and basic research has grown, but is still a start-off area in psychiatry when compared to other areas of medicine. The expectations of greater input into the classifications coming from neurobiological research will depend on the success of future initiatives like the Research Domain Criteria (RDoC) project, proposed by the National Institute of Mental Health (NIMH). The rationale of RDOC and DSM-5 are in opposite directions: the latter comes from the clinical observation of syndromes towards an understanding of their pathophysiology, and the former comes from the observation of basic neurobiological functions (or domains) towards an understanding of shared clinical features. ${ }^{12}$

\section{References}

1. Machado JD, Caye A, Frick PJ, Rohde LA. DSM-5. Major changes for child and adolescent disorders. In: Rey JM, editor. IACAPAP e-textbook of child and adolescent mental health. Geneva: International Association for Child and Adolescent Psychiatry and Allied Professions; 2013.

2. Kraemer HC, Kupfer DJ, Clarke DE, Narrow WE, Regier DA. How reliable is reliable enough? Am J Psychiatry. 2012;169:13-5.

3. Brotman MA, Schmajuk M, Rich BA, Dickstein DP, Guyer $A E$, Costello EJ, et al. Prevalence, clinical correlates, and longitudinal course of severe mood dysregulation in children. Biol Psychiatry. 2006;60:991-7.

4. Blader JC, Carlson GA. Increased rates of bipolar disorder diagnoses among U.S. child, adolescent, and adult inpatients, 1996-2004. Biol Psychiatry. 2007;62:107-14.

5. Leibenluft E. Severe mood dysregulation, irritability, and the diagnostic boundaries of bipolar disorder in youths. Am J Psychiatry. 2011;168:129-42.

6. Leibenluft E, Charney DS, Towbin KE, Bhangoo RK, Pine DS. Defining clinical phenotypes of juvenile mania. Am J Psychiatry. 2003;160:430-7.

7. Zepf FD, Holtman M. Disruptive mood dysregulation disorder. In: Rey JM, editor. IACAPAP e-textbook of child and adolescent mental health. Geneva: International Association for Child and Adolescent Psychiatry and Allied Professions; 2012.

8. Kieling C, Kieling RR, Rohde LA, Frick PJ, Moffitt T, Nigg JT, et al. The age at onset of attention deficit hyperactivity disorder. Am J Psychiatry. 2010;167:14-6.

9. Polanczyk G, Caspi A, Houts R, Kollins SH, Rohde LA, Moffitt TE. Implications of extending the ADHD age-of-onset criterion to age 12: results from a prospectively studied birth cohort. J Am Acad Child Adolesc Psychiatry. 2010;49:210-6.

10. DeSousa DA, Stringaris A, Leibenluft $E$, Koller SH, Manfro GG, Salum GA. Cross-cultural adaptation and preliminary psychometric properties of the Affective Reactivity Index in Brazilian Youth: implications for DSM- 5 measured irritability. Trends Psychiatry Psychother. 2013;35(3):171-80.

11. American Psychiatric Association. Diagnostic and Statistical Manual of Mental Disorders, 5th edition (DSM-5). Washington: APA; 2013.

12. Martin A, Volkmar FR, Lewis M. Lewis's child and adolescent psychiatry: a comprehensive textbook. Philadelphia: Lippincott Williams \& Wilkins; 2007. 